\title{
Quantum Quenching of Radiation Losses in Short Laser Pulses
}

\author{
C. N. Harvey, ${ }^{1, *}$ A. Gonoskov, ${ }^{1,2,3, \dagger}$ A. Ilderton, ${ }^{1,4, \$}$ and M. Marklund ${ }^{1, \S}$ \\ ${ }^{1}$ Department of Physics, Chalmers University of Technology, SE-41296 Gothenburg, Sweden \\ ${ }^{2}$ Institute of Applied Physics, Russian Academy of Sciences, Nizhny Novgorod 603950, Russia \\ ${ }^{3}$ Lobachevsky State University of Nizhni Novgorod, Nizhny Novgorod 603950, Russia \\ ${ }^{4}$ Centre for Mathematical Sciences, Plymouth University, PL4 8AA, United Kingdom
}

(Received 4 August 2016; revised manuscript received 26 November 2016; published 10 March 2017)

Accelerated charges radiate, and therefore must lose energy. The impact of this energy loss on particle motion, called radiation reaction, becomes significant in intense-laser matter interactions, where it can reduce collision energies, hinder particle acceleration schemes, and is seemingly unavoidable. Here we show that this common belief breaks down in short laser pulses, and that energy losses and radiation reaction can be controlled and effectively switched off by appropriate tuning of the pulse length. This "quenching" of emission is impossible in classical physics, but becomes possible in QED due to the discrete nature of quantum emissions.

DOI: 10.1103/PhysRevLett.118.105004

Continual advances in achievable laser power has spurred renewed interest in using intense light to study fundamental predictions of classical and quantum electrodynamics (QED) [1-5]. One cornerstone of such experiments is the collision of laser beams with particle bunches [6,7]. Particle motion in intense fields is inherently nonlinear, in particular due to radiation reaction (RR) which is the impact of energy loss on particle motion. RR can reduce collision energies [8], hinder particle acceleration schemes $[2,9,10]$, and is seemingly unavoidable. Much work has gone into demonstrating that RR, long thought negligible, must now be accounted for in order to accurately model state-of-the-art high intensity laser-matter interactions $[2,11,12]$. In contrast, we will show here that we can control, and effectively turn off RR by tuning the laser pulse length. We will also present a realizable experimental setup, requiring only modest parameters, with which to observe the effect and so demonstrate a possibility to control quantum processes in intense light-matter interactions.

Consider then the collision of an electron beam with an intense laser. The trajectory and energy evolution of the electrons follows, in QED, a probability distribution which is typically centred on lower energy losses than is predicted by classical physics. The reason is that while accelerated electrons must radiate continuously according to classical mechanics, the stochastic nature of quantum processes allows electrons to penetrate into the pulse before losing any significant energy to emission [13-15]. The purely quantum effect we present here is that electrons can interact with the entire laser pulse, but pass through it without losing energy to hard photons. This is forbidden in classical physics, where radiative losses and recoil effects are continuous phenomena $[2,11]$, but is made possible by tuning the laser pulse length and exploiting the discrete nature of quantum emission. Because of the latter, there is always a nonzero chance for the electrons to not emit any photons of sufficient energy to significantly backreact on the electron. To roughly estimate when this phenomena may be significant, we consider the elastic scattering probability $P_{0}$ that the electron does not emit radiation, $P_{0}=\exp \left(-P_{1}\right)$, where $P_{1}$ is the probability of one emission [16]. ( $P_{1}$ is infrared finite in laser backgrounds [17] and soft emission does not cause any significant backreaction on the electrons; see Supplemental Material [18] for details.) $P_{0}$ is exponentially damped with both intensity and pulse duration, so signatures of quantum effects, though present, are normally obscured in e.g. long laser pulses [28]; once the electrons emit they quickly lose energy, entering a classical regime. Thus in order to identify parameters for observing our effect we first consider short pulses.

In Fig. 1(a) we plot $P_{0}$ in a pulse of FWHM duration $2.7 \mathrm{fs}$, corresponding to one optical cycle [29], as a function of laser intensity and initial electron energy. The implied optimal parameters for intensity and energy are then verified in Fig. 1(b) where the electron energy loss is plotted as a function of pulse length. We simulate the laserparticle interactions using well-tested Monte Carlo routines [12,30,31]; see Supplemental Material [18]. As expected, the mean energy loss grows much more slowly in QED than classical physics predicts but, more significantly, the bright region in the lower left-hand corner of Fig. 1(b) shows that there is a high probability for the elections to pass through a short pulse without losing energy to emission.

Figure 2 shows the dynamics of a single relativistic electron passing through the center of an intense pulse. The upper panels show that the electron probability density is not centered on the radiating trajectory expected classically, but on the Lorentz force trajectory, i.e. a no-emission, norecoil path: even though the electron clearly interacts with, 
(a)

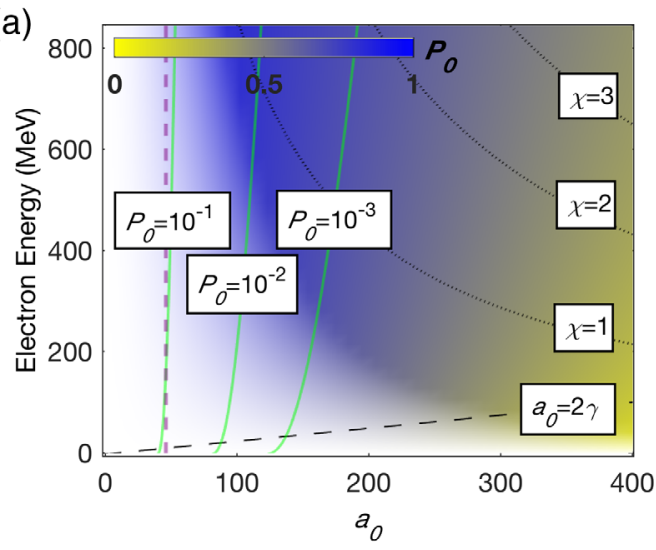

(b)

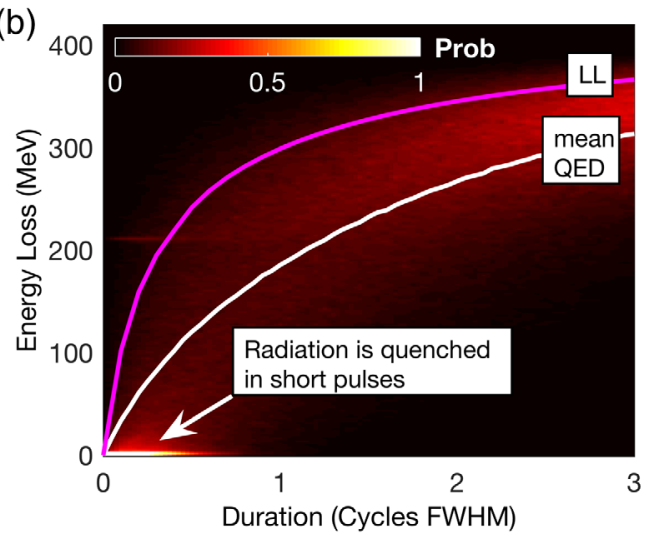

FIG. 1. (a) The no-emission probability $P_{0}$ in a one-cycle pulse as a function of initial electron energy and peak laser electric field $(E)$ in relativistic units $a_{0}=e E \lambda / 2 \pi m c^{2}$ (for electron charge $e$, mass $m$ and $c$ is the speed of light). The laser has wavelength $\lambda=0.82 \mu \mathrm{m}$ and spot size $w_{0}=5 \mu \mathrm{m}$. As the probability of not emitting is unity if, say, $a_{0}=0$ (no laser), we restrict attention to the radiation dominated regime [10,32] where the parameter $\epsilon_{\mathrm{rad}}=(2 / 3) \alpha \omega a_{0}^{2} \gamma / m \geq 1$ and significant recoil effects are expected: the color is therefore faded out for $\epsilon_{\mathrm{rad}}<1$. The optimal parameter region (blue) is then $a_{0} \in\{100 \ldots 200\}$ and electron energies in the 100s of MeV. This region overlaps with that where $\chi<1$ : we work throughout in this regime, so that pair production need not be considered [17]. Also shown is the line $a_{0}=2 \gamma$ above which classical RR causes reflection of the electron [33]. (b) Probability density of the electron energy loss in QED for initial electron energy $420 \mathrm{MeV}$ and $a_{0}=200$. The corresponding peak intensity is $1.1 \times 10^{23} \mathrm{~W} / \mathrm{cm}^{2}$, slightly beyond the state of the art [34] but within reach of upcoming facilities [35,36]. The average energy loss in QED (white line) grows more slowly than the classical prediction (pink line) of the Landau-Lifshitz equation [37] ("LL"; see Supplemental Material [18]). The bright yellowwhite region in the lower left-hand corner shows that there is a high probability for the elections to pass through a short pulse without losing energy to emission. This is "quenching."

and is accelerated by, the pulse, RR is effectively switched off.

We can make an analogy between this effect and that of chemical fluorescence quenching [38], in which excited electrons can move between molecules without emission of photons. This radiation-free transfer decreases the fluorescent intensity of a sample. Quenching mechanisms are typically short-range, with e.g. Dexter transfer being purely quantum mechanical and exponentially damped with distance [39]. Because of these similarities we refer to our effect as quenching of radiation. Quenching is related to "straggling" [13,15], that is the possibility for electrons to reach the focus of a laser pulse and emit higher energy photons than would be possible classically. Straggling is necessary but not sufficient for quenching: the latter requires the combination of the effects of straggling with a short duration pulse.

As a check we use known analytic results to recover some properties of Fig. 1. The invariant $\chi=$ $e \hbar \sqrt{p \cdot F^{2} \cdot p} / m^{3} c^{4}$ (where $F_{\mu \nu}$ is the electromagnetic field tensor and $p^{\nu}$ the electron momentum), parametrizes the importance of strong field quantum effects. These are present even at $\chi \ll 1$ [40], and in this regime the emission probability is well approximated by $d P_{1} / d t \simeq 1.44 \alpha \chi / m \gamma$ for $\alpha$ the fine structure constant $\gamma$ the electron gamma factor [17]. Given the high particle energy considered, it suffices to integrate over the plane-wave profile through the center of the pulse, which gives $P_{1} \simeq 1.44 \alpha a_{0} \times 4.69$, where $a_{0}$ is the peak field strength and the final numerical factor comes from integrating over the pulse profile (see Supplemental Material [18]). This implies that the curve $\exp \left(-P_{1}\right)=$ $1 / 10$ should be independent of $\gamma$, i.e. approximately vertical, at $a_{0} \simeq 47$, in excellent agreement with the plotted results; see the purple dashed line in Fig. 1(a).

With this confirmation, we can increase complexity by considering collisions between the laser and, now, a realistic electron bunch. Figure 3 shows the final distribution of electrons on a screen positioned $1 \mathrm{~mm}$ directly behind the laser. Parameters are chosen to optimize quenching: increasing $a_{0}$ widens the deflection angle of the classical electrons, while increasing $\gamma$ makes the final spot sizes smaller and more collimated. Classical predictions suggest that electrons radiate a substantial amount of their energy in the front tail of the pulse, causing them to slow down and be deflected. For longer durations, both the classical and quantum spectra are symmetric, though the latter exhibit typical stochastic spreading effects. For pulse durations of one cycle and below, the classical deflection is asymmetric. However, a quantum calculation shows that the electrons are now not deflected, but hit the center of the screen: quenching allows electrons to enter, and cross, field regions which are forbidden according to classical physics. This provides a clear signal which can be pursued experimentally: we look for electrons in places where there is zero classical background. (This same principle can also enhance signals of vacuum birefringence [41].) Note that, in contrast to straggling, we do not need to detect the emitted photons. 


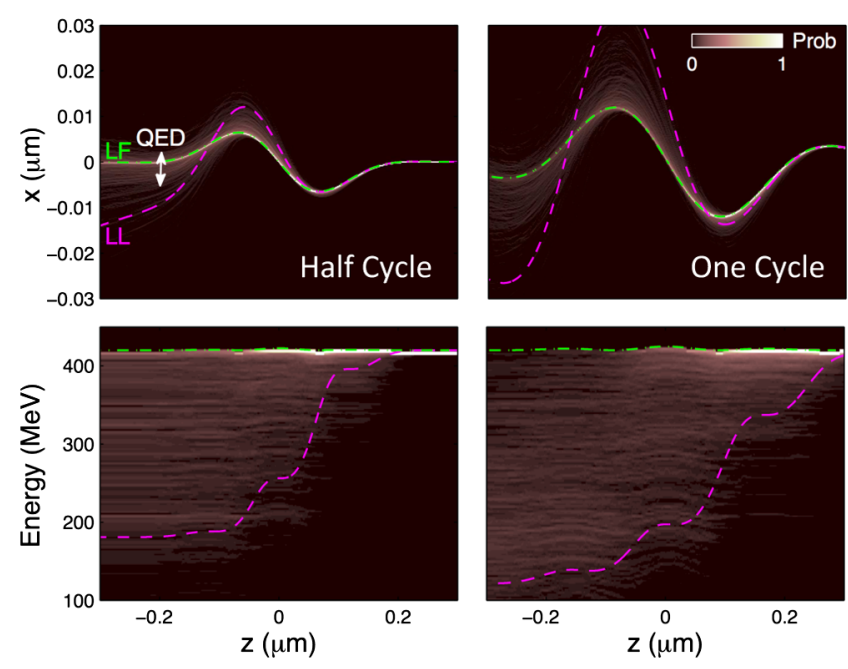

FIG. 2. Trajectories and energies of a single electron, incident from the right and passing through the center of an ultrashort, focused laser pulse of duration 0.5 cycles $(1.4 \mathrm{fs}$, left) and one cycle (2.7 fs, right). Other parameters as in Fig. 1(b). The colored region shows the QED probability distribution, calculated from 1000 simulations with the same initial conditions. (For clarity any region containing $>200$ electrons is colored as if it contained 200.) This distribution does not follow classical predictions which include RR, shown as a purple dashed line, but instead is visibly centered (white) on the Lorentz force curve shown with a dashed green line: this trajectory is by definition absent of RR. The lower panels show that, since the pulse is short, there is a high probability for the electrons not to emit until they are past the pulse peak. Even for the few electrons which subsequently emit, it is too late for their motion to be significantly affected.

Although the short pulses above are currently out of experimental reach, the stochastic nature of quantum emission means that quenching is still present even in longer pulses, although it is harder to observe because the electrons undergo classical-like cooling [43-45] before they emerge from the pulse. We therefore present a simple experimental setup, illustrated in Fig. 4(a), which filters out noise and mimics the short-pulse regime. (Note that field focusing will play an essential role here, in contrast to the parameters used for Fig. 1.) Here an electron bunch is brought into collision with a, now, tightly focused laser pulse. The electrons subsequently pass through a slit, then through a magnet, before being dumped onto a lanex screen. The setup is designed such that any electrons for which radiation is quenched will have properties distinct from all others and will populate a particular portion of the screen, free from noise. The laser is linearly polarized in the $x$ direction. The collision occurs along the $z$ axis. The magnet is orientated such that electrons are fanned out in the $y$ direction according to their energy, before hitting the screen. The main bulk of the electrons that miss the center of the laser focus will form a bright spot on the screen. Electrons passing close to the most intense part of the pulse can, for tight focusing, receive a significant deflection in
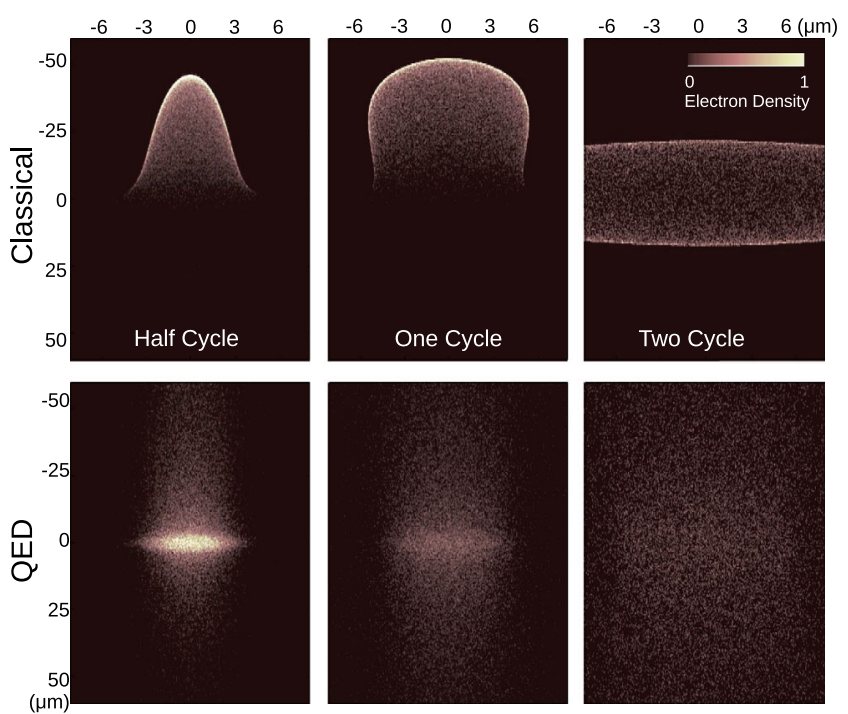

FIG. 3. Quenching in the collision of an electron bunch with laser pulses of different durations: the plots show the final electron distribution on a lanex screen $1 \mathrm{~mm}$ behind the focus. Classical predictions (top) show an asymmetric spatial distribution for short pulses. In the quantum results (bottom) the bright spot is formed by electrons which have traversed the pulse without significant emission or energy loss. The effect becomes less prominent as pulse length increases. For two cycles the classical distribution becomes symmetric, as one would expect, while in the quantum case stochastic transverse spreading [42] causes the bunch to spread out to a wider spot size than the classical bunch. The electron bunch initially had Gaussian distributions in energy, $420 \pm 0.35 \mathrm{MeV}$, and spacial position, $2 \mu \mathrm{m}$ FWHM. Laser intensity $a_{0}=200$, focal spot size $w_{0}=5 \mu \mathrm{m}$. (Parameters are similar to those achievable at ELI-NP [36].)

the $x$ direction. (This is larger than e.g. ponderomotive pushing could provide for electrons in the pulse periphery.) Electrons which have lost energy to emission will be fanned out by the magnet in the $y$ direction. However, electrons which have been significantly deflected in the $x$ direction but which have not been significantly deflected in $y$ have necessarily passed through the high-field region but, due to quenching, did not lose energy. The screen area they occupy cannot be populated by stochastic spreading, as all such electrons will have lost energy and will therefore be deflected in the $y$ direction. A possible source of noise would be electrons which have gained momentum in the $y$ direction due to either tight focusing effects, ponderomotive pushing, or stochastic spreading. These electrons would give a false reading, but are removed by the slit after exiting the laser, so never reach the magnet or screen.

Simulated experimental results are shown in Fig. 4(b) for parameters within reach of current facilities [36]. (The vector beam model used above has a ring singularity $[46,47]$; this was irrelevant for our previous parameters, but could cause problems for the wide electron beam used here. Therefore we use the particle-in-cell code ELMIS3D 

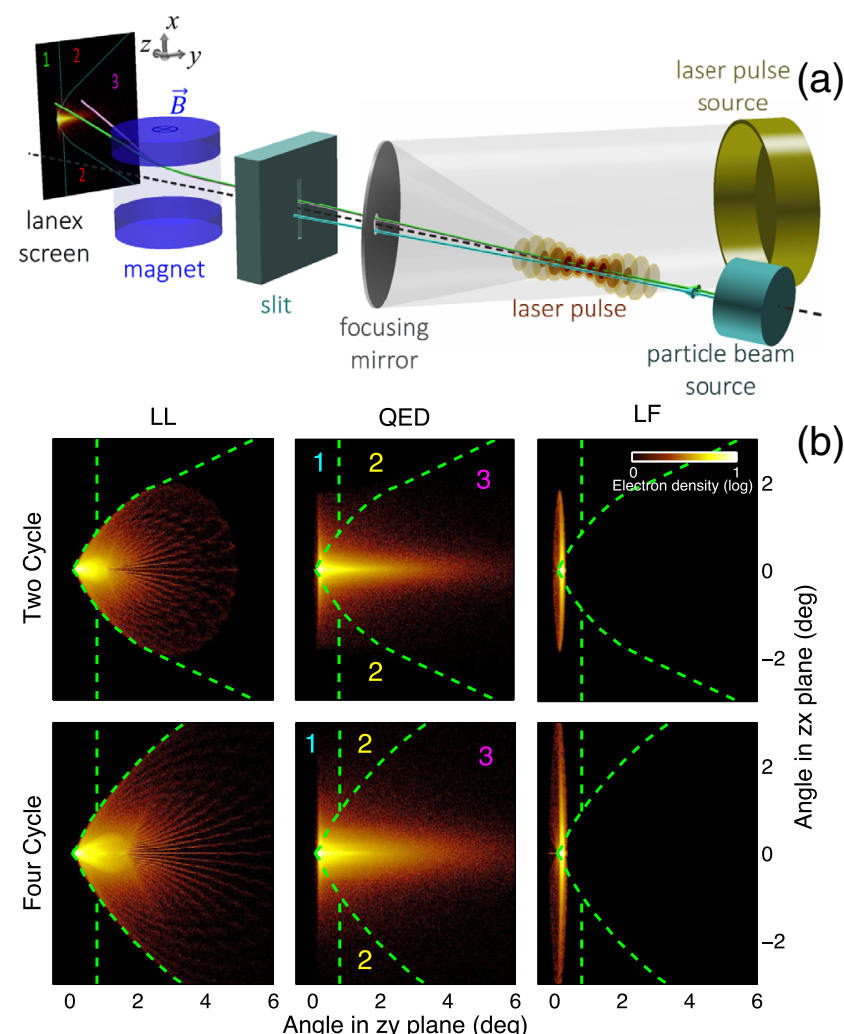

source

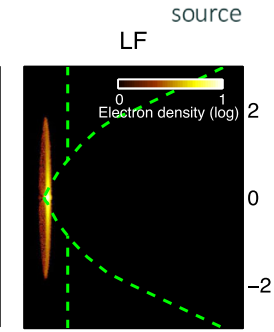

(b)

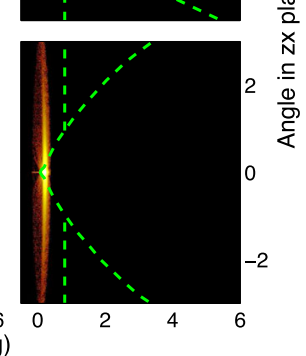

FIG. 4. (a) The proposed experimental setup for observing quenching. (b) Lanex screens for 2 and 4 cycle pulses. Peak intensity $a_{0}=50$ (50 TW total power), wavelength $820 \mathrm{~nm}$, tightly focused using an $f / 1$ optic. A $0.32 \mathrm{~T}, 15 \mathrm{~cm}$ magnet is placed $1 \mathrm{~m}$ behind the slit, which deflects electrons in the $y$ direction according to their energy. The lanex screen is placed $50 \mathrm{~cm}$ behind the magnet and angled at $45^{\circ}$. The electron beam energy is $100 \mathrm{MeV}$ (with $0.1 \%$ spread) and the beam is large enough so that precise synchronization would not be a concern in an experiment. The quantum distribution is very different from that predicted classically ("LL"), where electrons are confined to region 3. Quenched electrons, which have been deflected by the laser but not lost energy, will be delivered to region 1. Electrons can also appear in the nonclassical region 2, but due to stochastic spreading [42]: they have lost energy during the interaction. The boundary of region 1 is composed of the boundaries of the classical region 3 and the norecoil Lorentz force prediction ('LF").

[27] with QED routines described in Ref. [12].) The results demonstrate the feasibility of the proposal. The quantum prediction is vastly different to that of classical RR, signaling the presence of quantum effects, and the electron distribution more closely mimics the Lorentz force (no recoil) prediction. The electrons which have been deflected by the laser but not lost significant energy, demonstrating quenching, are deposited in region 1 of the screen. These electrons occupy an area of the screen which is free from classical noise. The electrons which have undergone quantum stochastic spreading hit the screen in region 2; they also occupy a nonclassical, but different, area of the screen. (The filamentation in the classical LL distribution is a

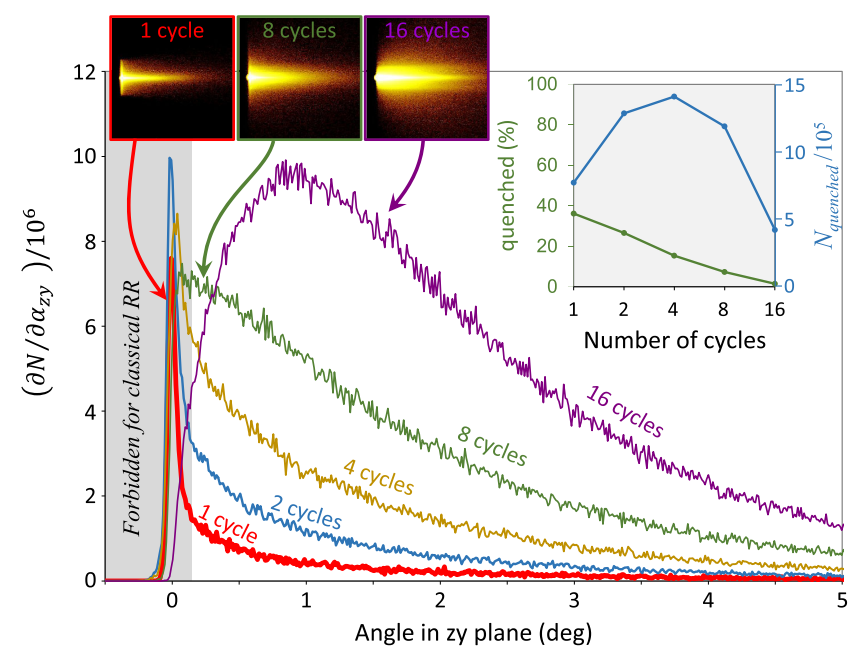

FIG. 5. 3D QED PIC simulation results showing the density of electrons deflected in the $x$ direction by $>0.5^{\circ}$ as a function of the $z y$ angle for pulse durations $1,2,4,8$, and 16 cycles (FWHM). The light gray zone $\left(<0.15^{\circ}\right)$ demarks the classically forbidden region. The upper right inset shows the dependency of the total number of quenched electrons (from the gray region) on the number of cycles (right-hand scale, blue), and as a percent of all particles deflected by more than $0.5^{\circ}$ in the $x$ direction (left-hand scale, green).

consequence of the tight pulse focusing.) Thus quenching can be observed even in longer laser pulses, and at currently available parameters. To underline this, Fig. 5 shows the proportion of electrons experiencing quenching as a function of pulse duration. Even for 16 cycles the number of quenched electrons is non-negligible $(\sim 1.5 \%)$. The detection of quenching is therefore within reach of existing facilities. The inset provides another experimental signature: a peak in the absolute number of electrons quenched as a function of pulse duration.

In conclusion, we have shown that quantum effects allow for an electron to be accelerated and decelerated by a short laser pulse without emitting hard photons; in effect radiation reaction is turned off, and electrons can follow Lorentz force trajectories, barely perturbed by emission and energy losses. One of the goals of new intense-laser facilities, such as ELI-NP, is to observe such fundamental quantum phenomena [36]. It is anticipated that high intensities and short pulse durations will come hand-inhand at future facilities [48], and indeed quenching is most prominent in short pulses. It can though also be observed in longer pulses using currently available parameters. Finally, we remark that determining the properties of high-intensity pulses remains an open and challenging problem: we show in the Supplemental Material [18] that quenching has a potential application here, as it can be used to measure the carrier envelope phase.

The authors are supported by the Knut \& Alice Wallenberg Foundation, the Swedish Research Council, 
Grants No. 2012-5644 and No. 2013-4248 (C. H. and M. M.), the Olle Engkvist Foundation, Grant No. 2014/ 744 (A. I.), the EU's Horizon 2020 research and innovation program under the Marie Skłodowska-Curie Grant No. 701676. (A. I.), the Russian Science Foundation, Project No. 16-12-10486 (A. G.), and the Russian Foundation for Basic Research Project No. 15-37-21015 (A. G.). A. I. thanks J. J. Phillips for useful discussions. Part of the simulations were performed on resources provided by the Swedish National Infrastructure for Computing (SNIC) and HPC2N.

*cnharvey@physics.org

†arkady.gonoskov@chalmers.se

*anton.ilderton@plymouth.ac.uk

\$mattias.marklund@chalmers.se

[1] M. Marklund and P. K. Shukla, Rev. Mod. Phys. 78, 591 (2006).

[2] A. Di Piazza, C. Muller, K. Z. Hatsagortsyan, and C. H. Keitel, Rev. Mod. Phys. 84, 1177 (2012).

[3] E. Lundstrom, G. Brodin, J. Lundin, M. Marklund, R. Bingham, J. Collier, J. T. Mendonca, and P. Norreys, Phys. Rev. Lett. 96, 083602 (2006).

[4] T. Heinzl, B. Liesfeld, K.-U. Amthor, H. Schwoerer, R. Sauerbrey, and A. Wipf, Opt. Commun. 267, 318 (2006).

[5] B. King, A. Di Piazza, and C. H. Keitel, Nat. Photonics 4, 92 (2010).

[6] S.-y. Chen, A. Maksimchuk, and D. Umstadter, Nature (London) 396, 653 (1998).

[7] C. Bula et al. (E144), Phys. Rev. Lett. 76, 3116 (1996).

[8] A. M. Fedotov, N. V. Elkina, E. G. Gelfer, N. B. Narozhny, and H. Ruhl, Phys. Rev. A 90, 053847 (2014).

[9] V. Malka, J. Faure, Y. A. Gauduel, E. Lefebvre, A. Rousse, and K. T. Phuoc, Nat. Phys. 4, 447 (2008).

[10] S. V. Bulanov, T.Z. Esirkepov, J. Koga, and T. Tajima, Plasma Phys. Rep. 30, 196 (2004).

[11] D. A. Burton and A. Noble, Contemp. Phys. 55, 110 (2014).

[12] A. Gonoskov, S. Bastrakov, E. Efimenko, A. Ilderton, M. Marklund, I. Meyerov, A. Muraviev, A. Sergeev, I. Surmin, and E. Wallin, Phys. Rev. E 92, 023305 (2015).

[13] C. S. Shen and D. White, Phys. Rev. Lett. 28, 455 (1972).

[14] R. Duclous, J. Kirk, and A. R. Bell, Plasma Phys. Controlled Fusion 53, 015009 (2011).

[15] T. G. Blackburn, C. P. Ridgers, J. G. Kirk, and A. R. Bell, Phys. Rev. Lett. 112, 015001 (2014).

[16] D. R. Yennie, S. C. Frautschi, and H. Suura, Ann. Phys. (N.Y.) 13, 379 (1961).

[17] V. I. Ritus, J. Sov. Laser Res. 6, 497 (1985).

[18] See Supplemental Material at http://link.aps.org/ supplemental/10.1103/PhysRevLett.118.105004, which contains references [17-27], for details of the simulation techniques and supporting analytical calculations.

[19] V. Dinu, C. Harvey, A. Ilderton, M. Marklund, and G. Torgrimsson, Phys. Rev. Lett. 116, 044801 (2016).

[20] N. V. Elkina, A. M. Fedotov, I. Yu. Kostyukov, M. V. Legkov, N. B. Narozhny, E. N. Nerush, and H. Ruhl, Phys. Rev. ST Accel. Beams 14, 054401 (2011).
[21] C. Ridgers, J. Kirk, R. Duclous, T. Blackburn, C. Brady, K. Bennett, T. Arber, and A. Bell, J. Comput. Phys. 260, 273 (2014).

[22] M. Vranic, J. Martins, R. Fonseca, and L. Silva, Comput. Phys. Commun. 204, 141 (2016).

[23] A. I. Nikishov and V. I. Ritus, Zh. Eksp. Teor. Fiz. 46, 776 (1964) [Sov. Phys. JETP 19, 529 (1964)].

[24] J. D. Jackson, Classical Electrodynamics (Wiley, New York, 1998).

[25] C. Harvey, T. Heinzl, and A. Ilderton, Phys. Rev. A 79, 063407 (2009).

[26] B. King, N. Elkina, and H. Ruhl, Phys. Rev. A 87, 042117 (2013).

[27] A. Gonoskov, Ph. D. thesis, Umeå University, 2013.

[28] C. N. Harvey, A. Gonoskov, M. Marklund, and E. Wallin, Phys. Rev. A 93, 022112 (2016).

[29] Q. Lin, J. Zheng, and W. Becker, Phys. Rev. Lett. 97, 253902 (2006).

[30] C. N. Harvey, A. Ilderton, and B. King, Phys. Rev. A 91, 013822 (2015).

[31] D. G. Green and C. N. Harvey, Comput. Phys. Commun. 192, 313 (2015).

[32] A. Di Piazza, Lett. Math. Phys. 83, 305 (2008).

[33] A. Di Piazza, K. Z. Hatsagortsyan, and C. H. Keitel, Phys. Rev. Lett. 102, 254802 (2009).

[34] V. Yanovsky, V. Chvykov, G. Kalinchenko, P. Rousseau, T. Planchon, T. Matsuoka, A. Maksimchuk, J. Nees, G. Cheriaux, G. Mourou, and K. Krushelnick, Opt. Express 16, 2109 (2008).

[35] C. Hernandez-Gomez, S. P. Blake, O. Chekhlov, R. J. Clarke, A. M. Dunne, M. Galimberti, S. Hancock, P. Holligan, A. Lyachev, P. Matousek, I. O. Musgrave, D. Neely, P. A. Norreys, I. Ross, Y. Tang, T. B. Winstone, B. E. Wyborn, and J. Collier, Central Laser Facility annual report, 2008-2009.

[36] I. Turcu, F. Negoita, D. Jaroszynski, P. Mckenna, S. Balascuta, D. Ursescu, I. Dancus, M. Cernaianu, M. Tataru, P. Ghenuche et al., Romanian Rep. Phys. 68, S145 (2016).

[37] L. D. Landau and E. M. Lifshitz, The Classical Theory of Fields, Course of Theoretical Physics, (Pergamon Press, Oxford, England, 1975), Vol. 2.

[38] L. Lackowicz, Principles of Fluorescence Spectroscopy (Springer, New York, 2006).

[39] D. L. Dexter, J. Chem. Phys. 21, 836 (1953).

[40] E. Nerush and I. Kostyukov, Nucl. Instrum. Methods Phys. Res., Sect. A 653, 7 (2011).

[41] F. Karbstein, H. Gies, M. Reuter, and M. Zepf, Phys. Rev. D 92, 071301 (2015).

[42] D. G. Green and C. N. Harvey, Phys. Rev. Lett. 112, 164801 (2014).

[43] I. Y. Pomeranchuk, Zh. Eksp. Teor. Fiz. 9, 915 (1939).

[44] N. Neitz and A. Di Piazza, Phys. Rev. Lett. 111, 054802 (2013).

[45] S. R. Yoffe, Y. Kravets, A. Noble, and D. A. Jaroszynski, New J. Phys. 17, 053025 (2015).

[46] A. M. Fedotov, K. Y. Korolev, and M. V. Legkov, Proc. SPIE Int. Soc. Opt. Eng. 6726, 672613 (2007).

[47] C. Harvey, M. Marklund, and A. R. Holkundkar, Phys. Rev. Accel. Beams 19, 094701 (2016).

[48] G. Mourou and T. Tajima, Science 331, 41 (2011). 